\title{
Preference and resistance to change in concurrent variable-interval schedules
}

\author{
MATTHEW C. BELL \\ Santa Clara University, Santa Clara, California \\ and \\ BEN A. WILLIAMS \\ University of California, San Diego, La Jolla, California
}

\begin{abstract}
Pigeons were trained on a multiple schedule in which separate concurrent schedules were presented in the two components of the schedule. During one component, concurrent variable-interval40-sec variableinterval 80 -sec schedules operated. In the second component, concurrent variable-interval 40 -sec variableinterval 20-sec schedules operated. After stable baseline performance was obtained in both components, extinction probe choice tests were presented to assess preference between the variable-interval 40-sec schedules from the two components. The variable-interval40-sec schedule paired with the variableinterval 80-sec schedule was preferred over the variable-interval40-sec schedule paired with the variableinterval 20-sec schedule. The subjects were also exposed to several resistance-to-change manipulations: (1) prefeeding prior to the experimental session, (2) a free-food schedule added to timeout periods separating components, and (3) extinction. The results indicated that preference and resistance to change do not necessarily covary.
\end{abstract}

Response strength is a theoretical construct that has been related to empirical measures in a variety of ways. Skinner (1938) proposed the concept of reflex reserve, which he measured by resistance to extinction. Later, after abandoning reflex reserve (e.g., Skinner, 1950), he invoked probability of responding as the measure of strength, with response rate serving as a surrogate for probability. Herrnstein (1970) proposed choice behavior as a more appropriate measure, on the basis of his demonstration of orderly relationships between choice behavior and reinforcement rates for concurrent schedules (the matching law), which he then extrapolated to simple and multiple schedules of reinforcement. Nevin (1974) proposed yet a third measure of response strength: resistance to change. Nevin (1974) defined response strength by using the concept of behavioral momentum, which implies that the strength of behavior is indexed by the persistence of responding in the presence of various disrupters (e.g., additional response-independent food). A critical issue for behavior analysis is to identify the relationshipbetween these different measures of response strength. Moreover, to the extent that they do not covary, which measure is more useful for determining the fundamental laws of behavior?

Portions of this research were presented at the 22nd annual convention of the Association for Behavior Analysis, San Francisco, and were carried out in partial fulfillment of the PhD requirements at UCSD for the first author. This research was supported by NSF grants to the University of California, San Diego, B.A.W., principal investigator. Correspondence concerning this article should be addressed to M. C. Bell, Santa Clara University, Psychology Department, 500 El Camino Real, Santa Clara, CA 95053 (e-mail: mbell@ scu.edu).
Dissociations between response rate and preference and between response rate and resistance to change have been demonstrated in studies of anticipatory behavioral contrast. Williams $(1991,1992)$ presented pigeons a four-component multiple schedule in which two target components (A and B) with identical reinforcement schedules were followed differentially by either extinction or a higher rate of reinforcement. Response rate was higher during Component A, which was followed by extinction. However, in probe tests in which the stimuli correlated with Component A and Component $B$ were presented together, preference was in favor of the stimulus correlated with Component B, which had been followed by the richer schedule. Thus, the increase in response rate produced by the contrast procedure was inversely related to the measure of preference. A similar dissociation between response rate and resistance to change was reported by Nevin, Smith, and Roberts (1987), who used a similar four-component schedule and then measured the degree of response persistence during various disruptors. Here, greater resistance to change occurred for the target component followed by the richer schedule.

On the basis of this and other evidence, Nevin (1979, 1992; Nevin \& Grace, 2000) has argued that resistance to change and preference are strongly, and perhaps perfectly, correlated measures of response strength and that both are more orderly indices of the effects of reinforcement training than is response rate. Nevin has further argued that the critical determinant of resistance to change is the rate of reinforcement in the presence of a stimulus, even when that reinforcement may not be contingent on operant responding in the presence of the stimulus. For example, Nevin, Tota, 
Torquato, and Shull (1990) arranged a two-component multiple schedule in which keypecking was reinforced according to the same variable-interval(VI) schedule in both components. Throughout baseline training, additional reinforcers were provided concurrently and independently of responding by a variable-time (VT) schedule in Component $\mathrm{A}$, but not in Component $\mathrm{B}$. Response rates were lower in Component A, consistent with many previous findings that the addition of response-independent reinforcement decreases response rate. However, resistance to change was greater in Component $\mathrm{A}$, indicating that the addition of response-independentreinforcement in the presence of a stimulus increased resistance to change.

Unfortunately, two key assumptions of behavioral momentum theory-that preference and resistance to change are interchangeable measures of response strength and that resistance to change is determined by the frequency of reinforcement in the presence of a stimulus-are seriously challenged by results from other experiments that have attempted to specify the controlling variables underlying the matching law. In an effort to assess the melioration theory of matching (see Williams, 1988, for a review), Williams and Royalty (1989) presented pigeons a multiple schedule in which concurrent VI 20-sec VI 120-sec schedules operated in Component A, whereas concurrent VI 60sec VI 80-sec schedules operated in Component B. The fundamentalassumption of the melioration theory of matching is that the obtained local rate of reinforcement is the primary determinant of choice and that matching results whenever the obtained local rates of reinforcement are equal for the different choice alternatives. Given that matching occurred in both components of the multiple schedule, the obtained local reinforcement rates during Component $\mathrm{A}$ were 210 reinforcers/ $h$ for both choice alternatives, whereas the local rates of reinforcement for both choice alternatives during Component B were 105 reinforcers/h. Thus, given a choice of the VI 120 -sec alternative from Component A versus the VI 60-sec alternative from Component $\mathrm{B}$, melioration theory predicts preference in favor of the VI 120-sec schedule. Behavioral momentum theory makes the same prediction, because the total rate of reinforcement in the presence of a stimulus is correlated with obtained local rates of reinforcement. The results of the preference tests were opposite this prediction: Preference was in favor of the VI 60-sec choice alternative. Similar results using discrete-trial probability schedules were reported by Williams (1993).

Conceptually similar experiments have been reported by Belke (1992) and Gibbon (1995), who used multiple schedules in which a concurrent VI 40-sec VI 80-sec schedule operated in one component and a concurrent VI 40 -sec VI 20-sec schedule operated in the other. When choice probe trials were presented involving the separate VI 40-sec schedules from the two components, preference was in favor of the VI 40-sec alternative paired with the VI 80 -sec alternative. Both melioration and behavioral momentum theory predict the opposite outcome, because both the obtained local rate of reinforcement and the total rate of reinforcement in the presence of the different VI 40-sec stimuli were higher for the VI 40-sec schedule paired with the VI 20-sec schedule. Thus, the Pavlovian association between a stimulus and the rate of reinforcement in its presence did not determine preference.

The results of the foregoing experiments imply either that preference and resistance to change are not interchangeable measures or that resistance to change, like preference, is not determined by the total rate of reinforcement in the presence of a stimulus. The present study is an attempt to determine which alternative is correct. The procedure reported by Belke (1992) is replicated, and both preference and resistance to change were assessed for the two VI 40-sec schedules.

\section{METHOD}

\section{Subjects}

Eight experimentally experienced pigeons (Columba livia) were maintained at $80 \%$ of their free-feeding body weights by additional feeding, when necessary, after each experimental session. Additional feeding occurred no sooner than $1 \mathrm{~h}$ after the experimental session. All were housed in individual cages, with water and grit freely available. Free-feeding weights were calculated by first providing all the pigeons with continuous access to both milo and pigeon chow for a period of at least 2 weeks prior to assessing free-feeding weights. All the birds then were weighed daily for 7 days. The data were used to calculate a mean free-feeding weight and an $80 \%$ weight goal. Pigeons' mean $80 \%$ weight was $459.6 \mathrm{~g}$, with a range of $397-533 \mathrm{~g}$.

\section{Apparatus}

Six subjects (W50, R17, R122, R151, R10, and R8) were studied in four identical cylindrical chambers $(36 \mathrm{~cm}$ high and $33 \mathrm{~cm}$ in diameter). Three response keys $(2 \mathrm{~cm}$ in diameter) were located $24 \mathrm{~cm}$ above the mesh floor and $7 \mathrm{~cm}$ apart, center to center. A force of approximately $0.10 \mathrm{~N}$ was required to operate the response keys. A white houselight was located $8 \mathrm{~cm}$ above the center key and was illuminated, except during reinforcer presentations. The food hopper opening $(5 \mathrm{~cm}$ high and $5 \mathrm{~cm}$ wide) was located $16 \mathrm{~cm}$ below the center key.

The remaining subjects (R124 and W6) were studied in identical, rectangular chambers. The chambers consisted of opaque black plastic side walls, sheet aluminum front and back walls, a plywood ceiling, and a wire mesh floor. Each chamber was $32 \mathrm{~cm}$ high, $35 \mathrm{~cm}$ wide, and $36 \mathrm{~cm}$ deep and had three response keys, each $2.5 \mathrm{~cm}$ in diameter, mounted $23 \mathrm{~cm}$ from the floor and $7.25 \mathrm{~cm}$ apart, center to center, on the front wall. Each key could be transilluminated from the rear and required a minimum force of approximately $0.15 \mathrm{~N}$ to operate. General chamber illumination was provided by a white houselight mounted $4 \mathrm{~cm}$ above the center key. Access to a solenoidoperated grain hopper, when activated, was available through a rectangular opening $5 \mathrm{~cm}$ high and $6 \mathrm{~cm}$ wide, located $9.5 \mathrm{~cm}$ below the center key.

For both chamber types, reinforcement was delivered via a solenoidoperated food hopper and consisted of 3-sec access to milo. While the hopper was raised, a white light illuminated it, and the keylights were extinguished. A ventilation fan was present in each chamber and continuously presented white noise to mask extraneous sounds. Experimental events and data recording were controlled by PC computers (programmed with Borland Turbo Pascal) located in an adjacent room.

\section{Procedure}

Baseline. Since all the pigeons were experienced, they were immediately placed in the baseline condition and exposed to a multi- 
ple schedule with alternating 90 -sec components, separated by a 60 sec timeout (TO) in which the houselight was illuminated and all keylights were dark and inoperative. Concurrent VI 40-sec VI 80sec schedules of reinforcement operated during 1 component of the multiple schedules, and concurrent VI 40-sec VI 20-sec schedules were in effect for the 2 nd component. The 2 components strictly alternated, with 10 of each type presented during daily sessions that lasted approximately $1 \mathrm{~h}$. All VI schedules were programmed using the Fleshler and Hoffman (1962) progression.

A diffuse yellow keylight on the center key was always correlated with the VI 20 -sec schedule. The VI 80 -sec schedule was always correlated with a white vertical line (on a dark background) on the center key. The two VI 40-sec schedules appeared on the side keys, red on the left key and green on the right. For 4 subjects (W50, R17, R10, and R8), the VI 40-sec schedule paired with the VI 20-sec alternative was correlated with a green keylight on the right key and the VI 40 -sec schedule paired with the VI 80-sec schedule was correlated with a red keylight on the left key. For the remaining subjects (R122, $\mathrm{R} 151, \mathrm{R} 124$, and W6), the color and position assignments for the two VI 40-sec schedules were reversed. Baseline sessions were in effect for 30 to 41 sessions, until relative response rates were judged to be stable by visual inspection.

Probe sessions. Probe sessions were identical to the previous baseline sessions, except that after every ninth baseline component, the subjects were presented with a 10-sec opportunity to respond to the stimuli correlated with the two VI 40-sec schedules (i.e., red on the left key and green on the right key), which were presented simultaneously. This method resulted in two probes' being presented each probe session and ensured that one probe would occur after a VI 40-sec VI 80-sec baseline component and the other would occur after a VI 40-sec VI 20-sec baseline component. During these probe trials, extinction was in effect on both response keys. Two baseline sessions occurred between each probe session. Eight probe sessions were completed. The subjects were then returned to the baseline condition for 10 sessions before proceeding to the prefeeding phase.

Prefeeding. Over consecutive days, all the pigeons were fed chow approximately $1 \mathrm{~h}$ prior to the beginning of each experimental session. The procedure was otherwise identical to the baseline condition. The pigeons were fed increasing amounts of chow over the duration of the prefeeding phase. For the first 2 sessions, the subjects were fed $20 \mathrm{~g}$, followed by 2 sessions in which they were fed $40 \mathrm{~g}$, followed by 10 sessions in which they were fed $60 \mathrm{~g}$ prior to the session. R124 was removed from the procedure after exposure to three 60 -g sessions because it had stopped responding.

Following the prefeeding phase, the pigeons were returned to $80 \%$ ad-lib weight. This was then followed by between 13 to 45 baseline sessions before the next phase.

VT 20-sec schedule of reinforcement. A VT 20-sec schedule was presented during the 1-min TO periods separating baseline components. Time spent receiving reinforcement was not included in timing the TO period. All other aspects of the procedure were identical to the baseline condition. Twenty-five sessions were conducted with the VT 20-sec schedule in operation. The pigeons were returned to $80 \%$ ad-lib weight and afterward returned to the baseline condition for 15 to 40 sessions.

VT 10-sec schedule of reinforcement. A VT 10-sec schedule was presented during the $1-\min$ TO periods between components, in the same fashion as the VT 20 -sec schedule condition. Fifteen sessions were conducted with the VT 10-sec schedule in operation. Again, the subjects were returned to $80 \%$ ad-lib weight and subsequently returned to the baseline condition for 16 to 45 sessions preceding the next phase.

Extinction. All schedules were changed to extinction for 11 to 15 sessions: All events remained identical to the baseline condition, except that the hopper and hopper light were inoperative.

\section{RESULTS}

\section{Baseline Performance}

Because there were no significant differences in the response rates for any of the four schedule components across the different baseline presentations, response rates calculated from the last 10 sessions of each baseline phase were averaged together. Considerable undermatching occurred in both concurrent schedules for the majority of subjects. The mean preference for the higher valued schedule was .57 for the concurrent VI 20-sec VI 40-sec and .62 for the concurrent VI 40-sec VI 80-sec. The corresponding relative rates of reinforcement were .65 and .68 . It should be noted that the undermatching that was obtained does not significantly impact the theoretical issue of the relation between preference and resistance to change.

Comparing the absolute response rates of the VI 40-sec schedules paired with different alternative schedules, the mean response rate for the VI 40-sec schedule paired with the VI 20-sec schedule [VI 40(20)] was 51.4 responses/min, whereas that for the VI 40-sec schedule paired with the VI 80 -sec schedule [VI 40(80)] was $76.7 \mathrm{responses} / \mathrm{min}$. This difference was statistically significant at the .05 criterion, which was used for this and subsequent statistical tests $[t(7)=4.1]$.

\section{Preference in Probe Sessions}

Figure 1 shows the results from the test sessions in which the stimuli correlated with the two VI 40-sec schedules were paired on occasional probe trials. Data presented in Figure 1 represent the mean preference for the VI 40(80) alternative from the eight probe sessions. Higher response rates occurred to the VI 40-sec schedule paired during training with the VI 80-sec alternative for 7 of the 8 subjects. The mean preference across subjects for VI 40(80) was .70, which was significantly different from indifference [0.5; $t(7)=3.4]$. The present results replicated those of the previous studies (e.g., Belke, 1992).

\section{Resistance to Change from Prefeeding}

Response rate to each stimulus during each resistanceto-change session was normalized relative to the baseline response rate for that stimulus, and the means of the normalized rates are shown in Figure 2A.

Response rate maintained by each of the four schedules regularly decreased as the amount of free food prior to the session was increased. As is shown in Figure 2A, the smallest rate of decrease occurred for the VI 20-sec schedule, and the most rapid decrease occurred for the VI 80-sec schedule. The critical comparison is between the two VI 40-sec schedules, which appear in Figure $2 \mathrm{~A}$ to be generally similar. A $2 \times 2 \times 4$ within-subjects analysis of variance (ANOVA), with component (VI 40 VI 20 or VI 40 VI 80 ), value (rich or lean schedule within a given component), and phase (baseline, prefeeding of 20, 40, or $60 \mathrm{~g}$ ) as factors, was used to analyze the resulting data. The main 


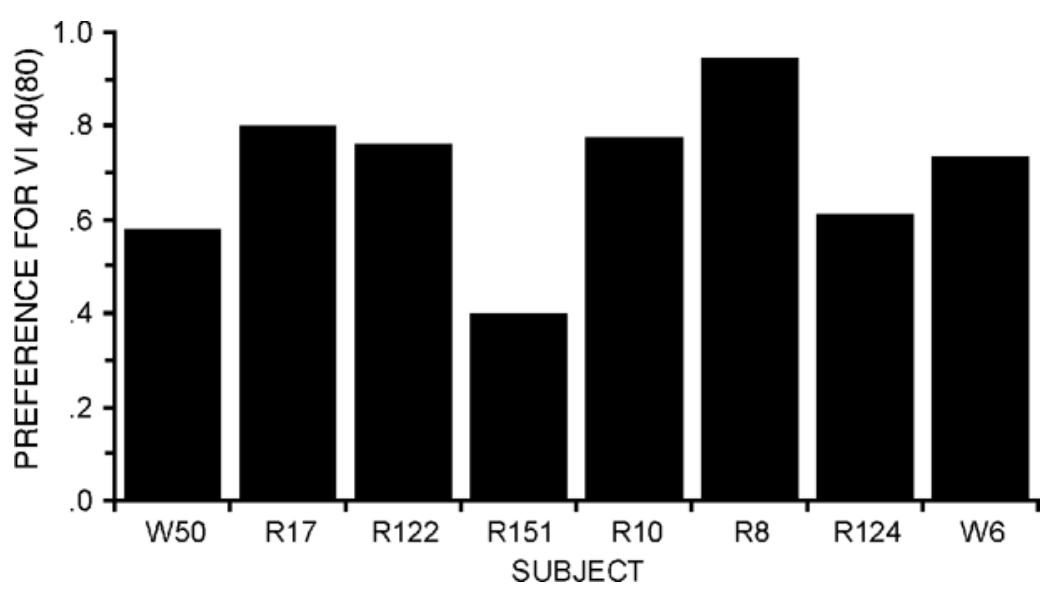

Figure 1. Relative response rates during extinction probe trials, showing preference for the stimulus correlated with the VI 40 -sec alternative that had been paired with the VI 80 -sec schedule (during baseline training) versus the VI 40 -sec alternative that had been paired with the VI 20 -sec schedule (during baseline training).

effect of phase and value were significant $[F(3,18)=13.8$ and $F(1,6)=6.8$, respectively], whereas the main effect of component was not significant, although it did approach statistical significance $[F(1,6)=5.6, p=.056]$. There were no significant interactions.

Nevin's (1992) theory of resistance to change implies that the total rate of responding in a component of a multiple schedule should be directly related to the total rate of reinforcement in that component. To examine this prediction, Figure 2B shows how the total response rate across both alternatives of each concurrent schedule was changed by the amount of prefeeding. Response rates decreased regularly in both components, but at a slower rate for the concurrent VI 20s VI 40s than for the concurrent VI 80s VI 40s.

Differential resistance to change for the choice alternatives within a concurrent schedule would imply that the choice proportion necessarily changes as a function of the resistance-to-change manipulation. Figure $2 \mathrm{C}$ plots the choice proportions in each component of the multiple schedule: Preference for the VI 40 over the VI 80 increased with amount of prefeeding, whereas preference for the VI 20 over the VI 40 also increased (as is shown by the decreasing slope of the lower function). Statistical analysis showed a significant trend (decreasing preference for the rich alternative) for the VI 40 VI 20 pair $[F(3,21)=5.1]$, but failed to produce a significant linear trend for the VI 40 VI 80 pair $[F(3,21)=2.9$; note, however, that it did approach significance, $p=.064]$.

\section{Resistance to Change From Interspersed Free Food}

Figure 3 plots the results from the phase of training in which a VT schedule of free food was presented in the 60sec periods interspersed between successive components of the multiple schedule. Figure $3 \mathrm{~A}$ shows that response rate was decreased substantially more for both schedules of the concurrent VI 40 VI 80 component than for the two schedules of the VI 40 VI 20 component. Unlike Figure 2A, there was no difference in the resistance to change between the individual schedules of a given concurrent pair. A $2 \times 2 \times 3$ within-subjects ANOVA, with component (VI 40 VI 20 or VI 40 VI 80), value (rich or lean schedule within a given component), and phase (baseline, VT 20, or VT 10) as factors, showed a significant main effect of component $[F(1,7)=12.9]$ and phase $[F(2,14)=6.5]$, but no significant effect of value $[F(1,7)<1]$. There was a significant component $\times$ phase interaction $[F(2,14)=6.6]$.

Because Figure $3 \mathrm{~A}$ indicates that no difference was evident between the resistance-to-change values of the different alternatives of either concurrent schedule, the expectation was that the choice proportions within each component should remain constant regardless of the VT value. Figure $3 \mathrm{C}$ shows that this was, in fact, the case.

\section{Resistance to Change From Extinction}

Figure 4A plots the decline of behavior for each choice alternative in each component of the multiple schedule. For all four functions, response rate during the fifth extinction session was less than $20 \%$ of the baseline values. The functions overlapped in different ways at different points in training, with no obvious relation between the slopes of the function and the schedule value.

Two separate analyses were conducted to examine the extinction data. Both data sets were analyzed using the proportion of baseline measure, but the first analysis included the baseline and first extinction session, whereas the second analysis simply excluded the baseline and the first extinction session. This adjustment was done to see whether there was a difference in outcome related to the argument that resistance to extinction involves two components: (1) disciminating the change in conditions and (2) resistance to change (Nevin \& Grace, 2000). 

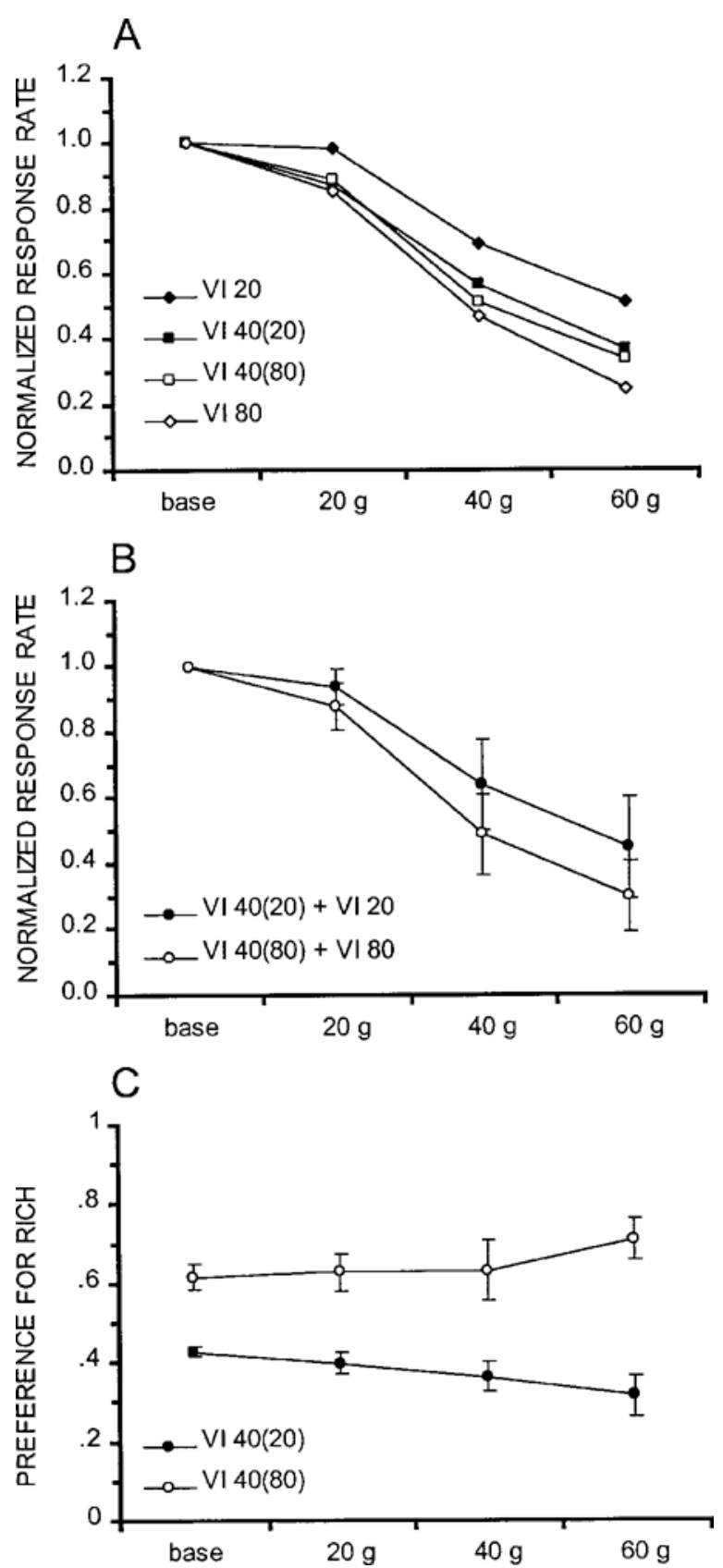

Figure 2. Prefeeding manipulation. Panel A shows the mean normalized response rate for all reinforcement schedules (both of the VI 40-sec schedules, the VI 20-sec schedule, and the VI 80 -sec schedule). Panel $B$ shows the mean normalized response rate for the sum of the two concurrent schedules (the sum of responding to the VI 20 -sec and the VI 40-sec schedules and the sum of responding to the VI 80-sec and the VI 40-sec schedules). Panel C shows preference for the rich alternative of each of the concurrent components of the multiple schedule. Error bars represent the standard error of the mean.

The first analysis (including all sessions) was a $2 \times 2 \times 7$ within-subjects ANOVA, with component(VI 40 VI 20 or VI 40 VI 80), value (rich or lean schedule within a given component), and session (baseline, Extinction Sessions 1,
$2,3,4$, or 5 , and the mean of the remaining sessions) as factors. It showed a significant main effect of session, $[F(6,42)=32.9]$. No other main effects or interactions were significant.

The second analysis (simply excluding the baseline and the first extinction session) was a $2 \times 2 \times 5$ within subjects ANOVA (again with component, value, and session as factors). There was a significant main effect of session $[F(4,28)=25.6]$ and a significant component $\times$ session interaction $[F(4,28)=4.3]$. There were no other significant main effects or interactions. Figure 4B shows the change in behavior, normalized to the baseline, for component responding and seems to suggest that the interaction is the result of the convergence of responding in the later stages of extinction as response rates approach zero.

Finally, Figure 4C shows the choice proportions within each component across the different extinction sessions. Although choice proportion for both components appears to be converging toward indifference, an ANOVA revealed no significant linear trends.

\section{DISCUSSION}

The purpose of the present study was to assess the apparent conflict between behavioral momentum theory and previous experiments addressing the melioration theory of the matching law. According to behavioral momentum theory, preference and resistance to change should be highly, if not perfectly, correlated; numerous experiments have reported a close correspondence between the two measures of response strength (e.g., Bell, 1999; Nevin \& Grace, 2000). A second assumption is that the main determinant of resistance to change is the rate of reinforcement that occurs in the presence of a stimulus, regardless of the response contingency associated with that reinforcement. At least some data (Bell, 1999) show this second assumption to be false, although the range of situations over which it fails remains unspecified. Previous experiments testing melioration theory suggest that these two assumptions cannot both be correct. In those experiments (Belke, 1992; Gibbon, 1995; Williams, 1993; Williams \& Royalty, 1989), two separate concurrent schedules were presented in a multiple schedule. For example, Belke presented a concurrent VI 40-sec VI 80-sec schedule in one component and a concurrent VI 40 -sec VI 20 -sec schedule in the alternative component of the multiple schedule. When preference between the two VI 40-sec schedules was assessed in separate probe trials, the VI 40-sec paired with the VI 80-sec alternative was strongly preferred, a finding opposite that predicted by behavioral momentum theory. Thus, either preference and resistance to change must be decoupled, or resistance to change for a given response alternative is not determined by the reinforcement in its presence.

The present study evaluated which of these possibilities was correct. We replicated the procedure of Belke (1992) and again demonstrated that preference on the probe trials favored the VI 40-sec paired with the lesser amount of alternative reinforcement (the VI 80 -sec schedule). After 

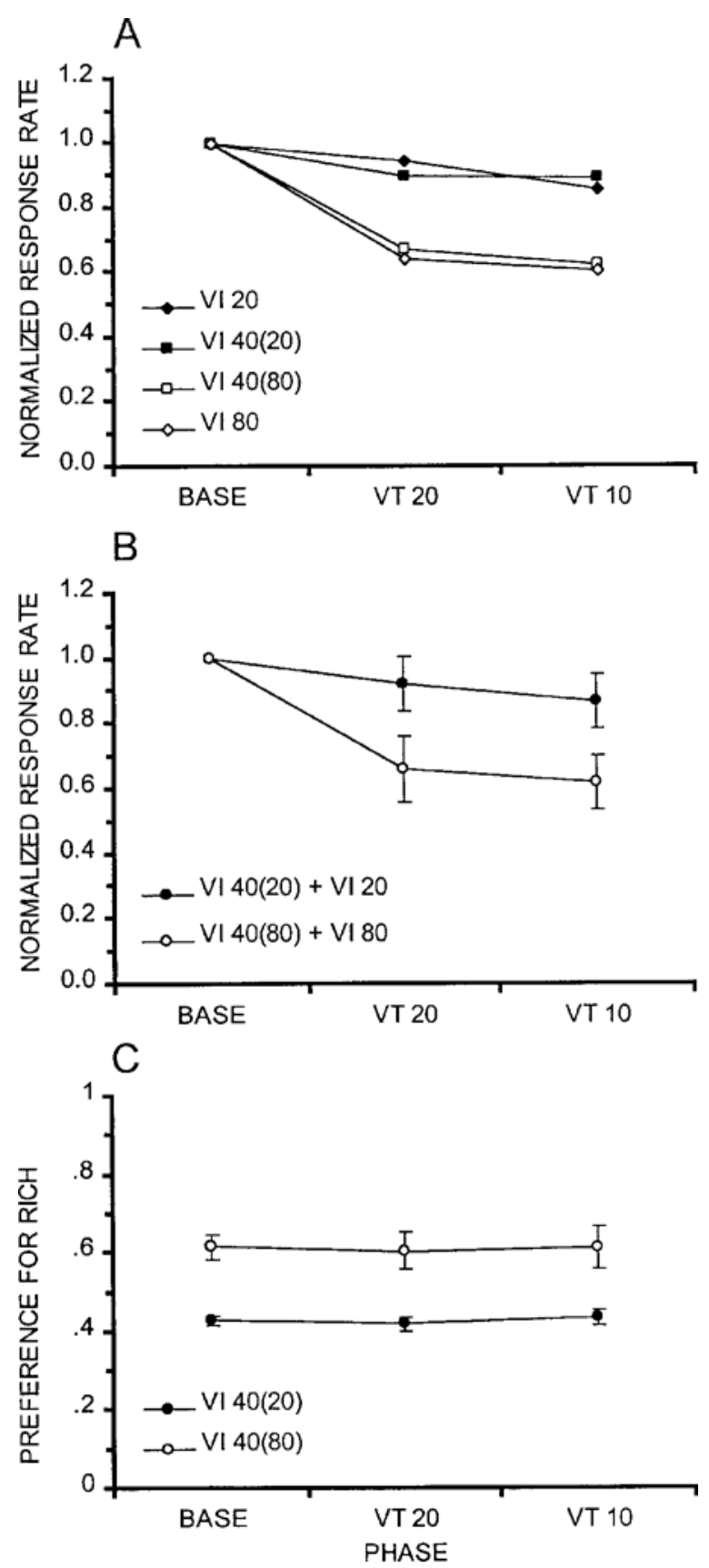

Figure 3. Free food manipulation. Panel A shows the mean normalized response rate for all reinforcement schedules (both of the VI 40-sec schedules, the VI 20 -sec schedule, and the VI 80-sec schedule). Panel $B$ shows the mean normalized response rate for the sum of the two concurrent schedules (the sum of responding to the VI 20-sec and the VI 40-sec schedules and the sum of responding to the VI 80-sec and the VI 40-sec schedules). Panel C shows preference for the rich alternative of each of the concurrent components of the multiple schedule. Error bars represent the standard error of the mean.

preference testing was completed, resistance to change was assessed by three different procedures: prefeeding, presentation of additionalfood between the components of the multiple schedule, and resistance to extinction.
The results of the different assessments of resistance to change were in general accord with previous findings, although somewhat variable for the different types of resistance-to-change assessments. When additional food was presented between the different components of the schedule, resistance to change was directly related to the rate of reinforcement from the different concurrent schedulesthat is, it was greater for both alternatives of the concurrent VI 40-sec VI 20-sec schedule. When resistance to change was assessed by different amounts of prefeeding before a session, resistance to change was again greater, in terms of total responding, for the component with the greater total reinforcement, although this effect was complicated by changes in the choice proportion. This was perhaps due to the considerable undermatching that occurred during the baseline training, for unknown reasons. When responding during extinction was used to assess resistance to change, it was necessary to exclude the first extinction session in order for a clear relation between resistance to change and reinforcement rate to emerge. This operation was anticipated by the earlier analysis of Nevin and Grace (2000), who argued that the effects of generalization decrement must be included in the analysis in order to account for the partial reinforcement extinction effect. Despite these complications, our results are generally consistent with previous findings regarding the relation between reinforcement rate and resistance to change (see McLean, Campbell-Tie, $\&$ Nevin, 1996, for results from a similar procedure). Thus, they allow us to address the major issue at hand: Why are resistance to change and preference inversely related, rather than being positively correlated, as postulated by behavioral momentum theory?

In their response to a preliminary report of the present data in our commentary (Williams \& Bell, 2000) on Nevin and Grace (2000), they argued that the apparent inverse relation between preference and resistance to change is an artifact of using an invalid assessment of preference. Their claim was that the type of choice probe tests we and others (Belke, 1992; Gibbon, 1995) have used is influenced by the carryover of response patterns from the concurrent schedule baseline training. They also suggested, instead, that the relative value of the two VI 40-sec components would be better assessed by arranging them as terminal links in concurrent chains to test the relative reinforcing strength in a manner used by Grace and Savastano (1997). Although that initial study produced ambiguous results, a subsequent study by Grace and Savastano (2000) has shown clearly that preference assessed in probe trials after training in concurrent chains is controlled by the absolute value of the terminal link schedules, regardless of the level of initiallink preference associated with them. For example, in Experiment 1 of their study, the initial-link schedules during Component A were VI 15-sec versus VI 15-sec with terminal links of VI 10-sec versus VI 20-sec; during Component B, the initial-link schedules were VI 30-sec versus VI 30 -sec, with terminal links of VI 20 -sec versus VI 40-sec. The results of probe trials using different combinations of the four different terminal links were determined entirely by the absolute values of the terminal-link schedules. 


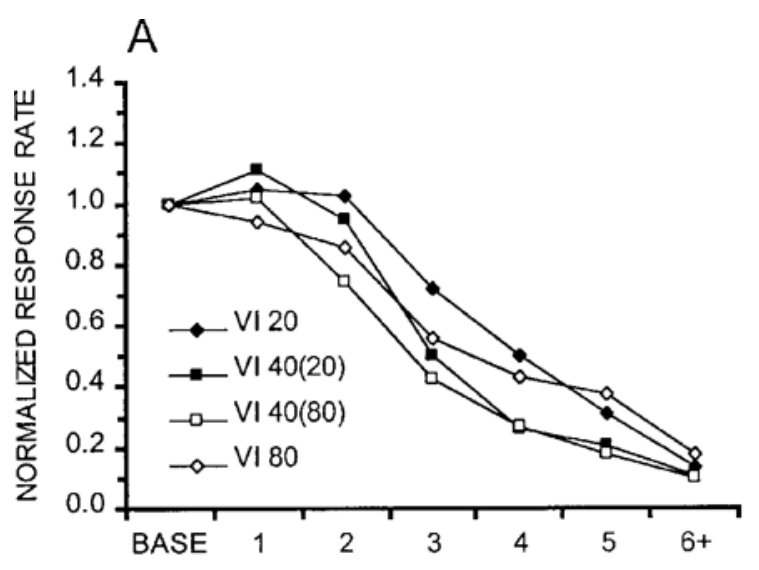

B
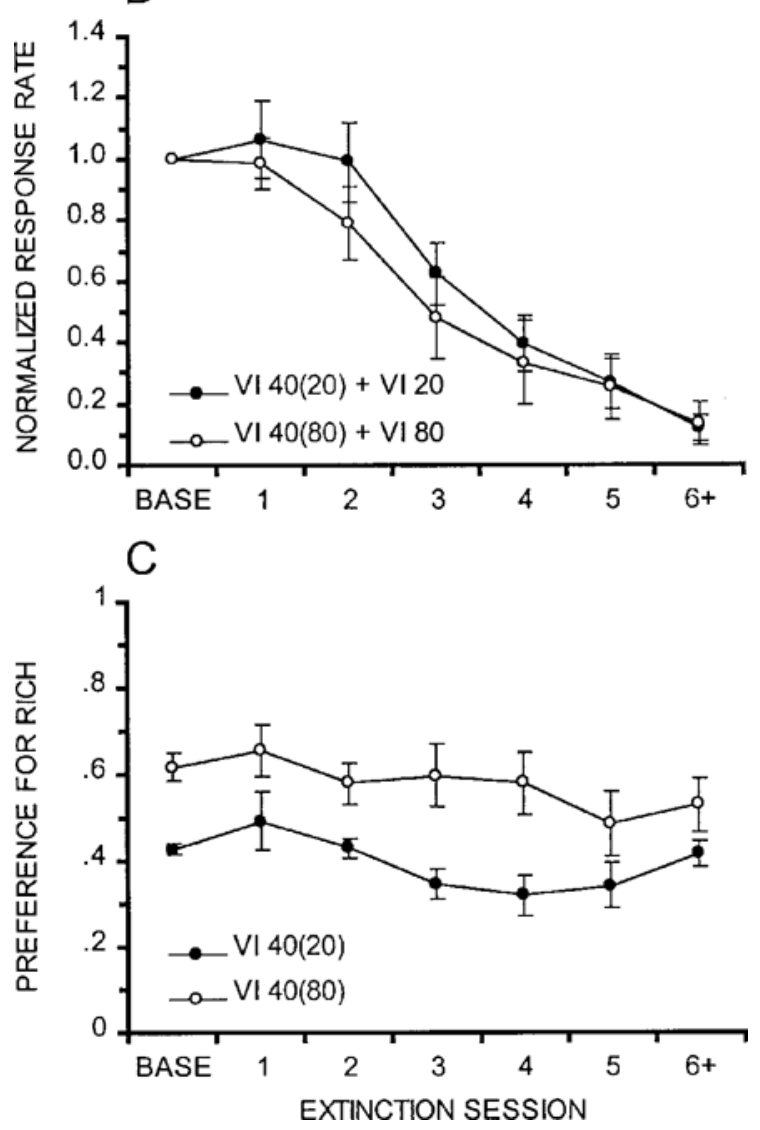

Figure 4. Extinction manipulation. Panel A shows the mean normalized response rate for all reinforcement schedules (both of the VI 40-sec schedules, the VI 20-sec schedule, and the VI 80-sec schedule). Panel B shows the mean normalized response rate for the sum of the two concurrent schedules (the sum of responding to the VI 20-sec and the VI 40-sec schedules and the sum of responding to the VI 80-sec and the VI 40-sec schedules). Panel C shows preference for the rich alternative of each of the concurrent components of the multiple schedule. Error bars represent the standard error of the mean.

We concur with Nevin and Grace (2000) that stimulus value assessed by probe preference tests after training with concurrent chains schedules is determined by the absolute value of the terminal-link schedules, but this ap- plies only to the terminal-link stimuli, not to the initiallink stimuli, which are most directly relevant to the relation between preference and resistance to change after training with simple concurrent schedules. As was noted by Nevin (1992), the predictions of behavioral momentum theory for concurrent schedules are identical to the melioration theory of matching. Given that melioration theory assumes that the local rate of reinforcement, not the actual schedule value, determines the value of a choice alternative, the determinants of the value of the terminallink stimuli of concurrent chains is orthogonal to the issue of the determinants of value of the different alternatives of a choice procedure.

Regardless of the determinants of stimulus value in concurrent chains procedures, the critical issue for the present study is how behavior during the probe tests after training on simple concurrent schedules should be interpreted. Does the probe trial behavior simply reflect the pattern of changeover behavior established during baseline training, or does it instead provide a meaningful measure of stimulus value distinct from the carryover effects? Several different experiments bear importantly on this issue.

In apparent support of the carryover interpretation are the results of Williams and Bell (1996), who replicated the procedure of Belke (1992), but with the variation that the VI 20-sec schedule was changed to a signaled VI 20-sec schedule. This change in the stimulus conditions had little effect on the rate of reinforcement produced by the schedule but radically reduced the response rate, so that little responding occurred except when the signal was present. The result was that responding was primarily to the unsignaled VI 40-sec schedule paired with the signaled VI 20 -sec schedule. Then, when the two VI 40-sec schedules (both unsignaled) were paired on probe trials, preference was strongly in favor of the VI 40-sec schedule that had been paired in training with the signaled VI 20-sec schedule, a result opposite to that when the VI 20 -sec schedule was unsignaled.

Although the results of Williams and Bell (1996) are consistent with a carryover interpretation, they are also consistent with an interpretation in terms of stimulus control of the probability of reinforcement. During probe trials, the signal from the signaled VI 20-sec schedule during baseline training was never present. Because extinction had been in effect on the alternative response key in the absence of the signal during training, this implies that the value of the VI 40-sec schedule during the probe trials was possibly the same as the value of the VI 40-sec schedule during baseline when the alternative schedule was extinction, which presumably was greater than the relative value of the alternative VI 40 -sec schedule that was paired with the unsignaled VI 80-sec schedule.

Evidence that simple carryover to the probe trials of the pattern of changeovers established during baseline training is an inadequate explanation of probe trial responding is provided by Williams and Royalty (1989, Experiment 1), who presented a concurrent VI 60-sec VI 80-sec schedule in one component of a multiple schedule and a concurrent VI 20-sec VI 120-sec schedule in the second component. 
Probe preference tests were then presented for the VI 60$\mathrm{sec}$ versus VI 120-sec alternatives and for the VI 20-sec versus VI 80 -sec alternatives. The average preference during baseline training was .56 for the VI 60-sec alternative, whereas it was .86 for the VI 20 -sec alternative. Assuming that probe trial responding was determined by the stay durations established during baseline training and that the average stay durations for a given schedule alternative was correlated with its relative response rate during baseline training, preference on the probe trials should be predicted by the ratio of the relative response rates for the alternatives paired on the probe trials. Thus, for the VI $60-\mathrm{sec}$ versus VI 120-sec probes, the relative value of the stay duration is predicted by the preference during baseline training for the VI 60 -sec ( .56) versus the preference for the VI 120 -sec (.14), or a value of 4.0. For the VI 20 -sec versus VI 80 -sec probe trials, the corresponding preferences during baseline training were .86 versus .43 , or a ratio of 2.0. Thus, preference during the probe trials should have greater for the VI 60 versus the VI 120 than for the VI 20sec versus VI 80-sec. In fact, however, preference for the VI 60-sec was .52, whereas preference for the VI 20-sec was .82. Thus, the relative durations of the stay times established during baseline training did not correctly predict the results during probe testing.

More direct evidence against the carryover interpretation of probe trial responding comes from Williams and Bell (1999), who explicitly manipulated the pattern of changeover behavior while holding the reinforcement schedules constant. Concurrent VI 30-sec VI 60-sec schedules were presented in both component of a multiple schedule. During one component, short changeoverdelays (CODs) were used, which produced short visit times to each alternative. The second component used long CODs that produced substantially longer visit times. When probe tests pitted the two VI 30-sec schedules against each other or the two VI 60-sec schedules against each other, simple transfer of the baseline pattern of behavior would predict preference for the schedule associated with the longer CODs during baseline training. In fact, indifference was observed, presumably because the choice was between schedules of equal value. Moreover, when the VI 30-sec schedule trained with a short COD was paired with the VI 60 -sec schedule trained with the long COD, or vice versa, preference was similarly in favor of the VI 30 -sec schedule regardless of the COD values used in training.

The notion that our probe trial preference results are transfer artifacts rather than valid measures of the relative value of the schedules is further challenged by the results of Williams (1993), who used a discrete-trial rather than a free-operant procedure (also see Williams \& Royalty, 1989, Experiments 2 and 3 ). This procedure entailed a single response on each trial, with an intertrial interval of several seconds separating trials. Thus, the notion that a pattern of changeover behavior transferred from baseline training to probe testing can be applied only with great difficulty. In that study, one component of a multiple schedule presented a choice between the left and the right response keys, one of which was associated with a probability of re- inforcement of .20 , whereas the other was associated with a probability of .05 . Reinforcers once scheduled were held until the next response to that alternative, so that the discretetrial contingencies were formally analogous to concurrent VI VI schedules. The subjects approximately matched their choice proportions to the obtained reinforcement proportions. In the second component of the multiple schedule, only the center key was available, which was associated with a reinforcement probability of .10 .

Behavioral momentum theory predicts that resistance to change should have been the same for both alternatives of the concurrent schedule, because the total rate of reinforcement in the presence of each alternative was the same. Assuming that resistance to change and preference are strongly correlated, probe tests in which one of the alternatives from the concurrent schedule was pitted against the response alternative with the constant .10 reinforcement probability should have produced the same outcome regardless of which alternative from the concurrent schedule was used in the probe test. In fact, however, the .20 alternative was strongly preferred over the .10 schedule, whereas the .10 schedule was strongly preferred over the .05 alternative. Thus, not only were the values of the two alternatives of the concurrent schedule different, those values were predicted by the schedule associated with the specific alternative, not the total reinforcement summed over both alternatives. It should be noted, however, that no resistance to change tests have been conducted after training with discrete-trial reinforcement procedures, so it is unclear whether such an assessment would yield results parallel to the preference results.

The proper interpretation of the results of probe trials like those used here and in earlier studies goes beyond the issue of the relationship between preference and resistance to change. The use of the probe procedure was motivated initially by the goal of testing the melioration theory of the matching law, which assumes that the local (recently obtained) rate of reinforcement per unit time responding, not the molar rate of reinforcement based on the schedule value, is the underlying variable controlling choice. If probe tests are not valid tests of the value of a given choice alternative, results that apparently strongly challenge melioration theory (and related theories) must be set aside (e.g., Williams, 1994). It should be noted, however, that melioration theory has been significantly challenged by several other types of evidence (Mark \& Gallistel, 1994; Myerson \& Hale, 1988; Rachlin \& Baum,1972) and that the probe trial procedure has proven highly useful after training with other procedures (Vom Saal, 1972; Williams, 1991).

Quite apart from the present findings, there are substantial reasons for challenging behavioral momentum theory as applied to choice procedures. The assumption that resistance to change is determined by the rate of reinforcement present during a given discriminative stimulus can be questioned on several grounds. Already noted in the introduction are the contrary results when reinforcement is presented after unsignaled delays of reinforcement (Bell, 1999). More generally, the notion of being in the presence 
of a stimulus is ill defined. Consider, for example, a concurrent schedule using a changeover key procedure in which the different choice alternatives are never simultaneously present but, instead, are alternated whenever an explicit changeover response occurs. Are the reinforcers presented during one of the schedule alternatives in the presence of the stimulus associated with the schedule alternative? If so, how does the changeover-key concurrent schedule differ from a multiple schedule that uses very short components that rapidly alternate? Given the similarity in the obtained relative rate of responding in such a procedure (Killeen, 1972), the notion that the concurrent schedule procedure and the multiple schedule procedure have qualitatively different determinants seems unlikely to be valid.

\section{REFERENCES}

BELKe, T. W. (1992). Stimulus preference and the transitivity of preference. Animal Learning \& Behavior, 20, 401-406.

BELL, M. C. (1999). Pavlovian contingencies and resistance to change in a multiple schedule. Journal of the Experimental Analysis of Behavior, 72, 81-96.

Fleshler, M., \& Hoffman, H. S. (1962). A progression for generating variable-interval schedules. Journal of the Experimental Analysis of Behavior, 5, 529-530.

GibBon, J. (1995). Dynamics of time matching: Arousal makes better seem worse. Psychonomic Bulletin \& Review, 2, 208-215.

Grace, R. C., \& Savastano, H. I. (1997). Transfer tests of stimulus value in concurrent chains. Journal of the Experimental Analysis of Behavior, 68, 93-115.

Grace, R. C., \& Savastano, H. I. (2000). Temporal context and conditioned reinforcement value. Journal of the Experimental Psychology: General, 129, 427-443.

Herrnstein, R. J. (1970). On the law of effect. Journal of the Experimental Analysis of Behavior, 13, 243-266.

KilleEN, P. (1972). A yoked-chamber comparison of concurrent and multiple schedules. Journal of the Experimental Analysis of Behavior, 18, 13-22.

Mark, T. A., \& Gallistel, C. R. (1994). The kinetics of matching. Journal of Experimental Psychology: Animal Behavior Processes, 20, 79-95.

McLean, A. P., CAmpbell-Tie, P., \& Nevin, J. A. (1996). Resistance to change as a function of stimulus-reinforcer and location-reinforcer contingencies. Journal of the Experimental Analysis of Behavior, 66, 169-192.

Myerson, J., \& Hale, S. (1988). Choice in transition: A comparison of melioration and the kinetic model. Journal of the Experimental Analysis of Behavior, 49, 291-302.

Nevin, J. A. (1974). Response strength in multiple schedules. Journal of the Experimental Analysis of Behavior, 21, 389-408.
NeVIn, J. A. (1979). Reinforcement schedules and response strength. In M. D. Zeiler \& P. Harzem (Eds.), Reinforcement and the organization of behavior (pp. 117-158). New York: Wiley.

Nevin, J. A. (1992). An integrative model for the study of behavioral momentum. Journal of the Experimental Analysis of Behavior, 57, 301-316.

Nevin, J. A., \& Grace, R. C. (2000). Behavioral momentum and the law of effect. Behavioral \& Brain Sciences, 23, 73-130.

Nevin, J. A., Smith, L. D., \& Roberts, J. (1987). Does contingent reinforcement strengthen operant behavior? Journal of the Experimental Analysis of Behavior, 48, 17-33.

Nevin, J. A., Tota, M. E., Torquato, R. D., \& Shull, R. L. (1990). Alternative reinforcement increases resistance to change: Pavlovian or operant contingencies? Journal of the Experimental Analysis of Behavior, 53, 359-379.

RACHLIN, H., \& BAUM, W. M. (1972). Effects of alternative reinforcement: Does the source matter? Journal of the Experimental Analysis of Behavior, 18, 231-241.

SkINner, B. F. (1938). The behavior of organisms. Acton, MA: Copley Publishing Group.

Skinner, B. F. (1950). Are theories of learning necessary? Psychological Review, 57, 193-216.

Vom SAaL, W. (1972). Choice between stimuli previously presented separately. Learning \& Motivation, 3, 209-222.

Williams, B. A. (1988). Reinforcement, choice, and response strength. In R. C. Atkinson, R. J. Herrnstein, G. Lindzey, \& R. D. Luce (Eds.), Stevens' Handbook of experimental psychology: Vol. 2. Learning and cognition (2nd ed., pp. 167-244). New York: Wiley.

Williams, B. A. (1991). Behavioral contrast and reinforcement value. Animal Learning \& Behavior, 19, 337-344.

WiLliams, B. A. (1992). Inverse relations between preference and contrast. Journal of the Experimental Analysis of Behavior, 58, 303-312.

Williams, B. A. (1993). Molar versus local reinforcement probability as determinants of stimulus value. Journal of the Experimental Analysis of Behavior, 59, 163-172.

Williams, B. A. (1994). The role of probability of reinforcement in models of choice. Psychological Review, 101, 704-707.

Williams, B. A., \& Bell, M. C. (1996). Changeover behavior and preference in concurrent schedules. Journal of the Experimental Analysis of Behavior, 65, 513-526.

Williams, B. A., \& Bell, M. C. (1999). Preference after training with differential changeover delays. Journal of the Experimental Analysis of Behavior, 71, 45-55.

Williams, B. A., \& Bell, M. C. (2000). The uncertain domain of resistance to change. Behavioral \& Brain Sciences, 23, 116-117.

Williams, B. A., \& Royalty, P. (1989). A test of the melioration theory of matching. Journal of Experimental Psychology: Animal Behavior Processes, 15, 99-113.

(Manuscript received March 20, 2000; revision accepted for publication October 16, 2001.) 